\title{
Predicting the effect of interfacial flow of insoluble surfactant on the deformation of drops rising in a liquid
}

\author{
$\begin{array}{ll}\text { M. R. Davidson } & \text { D. J. E. Harvie } \\ \end{array}$
}

(Received 29 August 2006; revised 11 December 2007)

\begin{abstract}
The volume-of-fluid numerical method of Rudman is extended to incorporate interfacial flow of insoluble surfactant using a published volume-of-fluid-based algorithm for surfactant transport. The extended technique is used to predict the transient axi-symmetric deformation of a drop rising in a liquid column. Predictions of flow and deformation with and without surfactant are compared and discussed. The results demonstrate the viability of the extended Rudman method for simulating interface dynamics in two-fluid flow which includes surface tension driven flow due to surfactant transport on the interface.
\end{abstract}

See http://anziamj.austms.org.au/ojs/index.php/ANZIAMJ/article/view/108 for this article, (c) Austral. Mathematical Soc. 2007. Published December 19, 2007. ISSN 1446-8735 


\section{Contents}

1 Introduction

C662

2 Formulation

C663

3 Results and discussion

C666

4 Conclusion

C672

References

C672

\section{Introduction}

Predicting the deformation of fluid interfaces such as drops, bubbles, or free surfaces is important in a wide range of applications. When surfactant is present, the surface tension is reduced depending on the surface concentration of surfactant; this modifies the interface deformation behaviour. Fluid flow redistributes the surfactant along the interface. This not only results in a non-uniform surface tension but also sets up a surface tension driven flow due to Marangoni forces [13] which oppose the surfactant redistribution. Both effects influence the surface deformation.

Most numerical studies of Marangoni effects on drop deformation due to surfactant concern drops in simple shear or extensional flow when the surfactant is insoluble. Stokes flow boundary integral methods are a popular choice to simulate such effects on drop deformation [1, 9, 14, 16, 21, 22]; finite volume [1], finite element [11], and immersed interface [12] methods have also been used to simulate such effects. Volume-of-Fluid (VOF) methods that include insoluble surfactant transport have also been developed recently by Renardy and coworkers [17, 8] and by James and Lowengrub [10]; these methods were applied to drop deformation under shear and extension, respectively. 
A range of methods have also been used to predict other interfacial flows such as bubble burst (potential flow boundary integral [3]) and deforming liquid bridges (finite element [15]).

VOF methods are well suited to describe large interface deformations including changes to the interface topology (for example, breakup or merging of drops). They capture the interface location on a fixed grid by advecting a scalar indicator (for example, the volume function of one phase) with the velocity field using special techniques designed to confine the spread of the numerical 'interface' [20]. Davidson, Rudman and Stevens [6, 7] used the Rudman VOF algorithm [19], adapted to include mass transfer in each phase, to simulate a range of experiments with rising/falling drops, but ignored possible Marangoni effects by assuming a uniform interfacial tension. In practice, liquids contain surface active contaminants, or have surfactant added to reduce the overall interfacial tension, in which case Marangoni forces may be important. Marangoni flows caused by surface concentration gradients of surfactant can reduce the internal circulation in a rising drop or bubble, making it behave more like a solid sphere and so reducing the rise velocity. We now extend the Rudman algorithm to include the vof-based technique for surfactant transport developed by James and Lowengrub [10]. The aim of this article is to demonstrate the extended algorithm by applying it to a drop, covered with insoluble surfactant, rising in a liquid column. The predicted drop deformation when there is surfactant transport is compared with predictions when there is no surfactant transport, and the reasons for any differences are discussed in section 3 .

\section{Formulation}

Consider a drop of radius $a$ rising in a column of immiscible liquid when the interface of the drop is covered with insoluble surfactant. 
The dimensionless equations of motion for a VOF flow calculation are

$$
\begin{aligned}
\frac{\partial C}{\partial t}+\nabla \cdot(\mathbf{U} C) & =0 \\
\frac{\partial \rho \mathbf{U}}{\partial t}+\nabla \cdot(\rho \mathbf{U U}) & =-\nabla P+\frac{1}{\mathrm{Fr}} \rho \hat{\mathbf{g}}+\frac{1}{\mathrm{We}} \mathbf{F}_{\mathbf{S}}+\frac{1}{\operatorname{Re}} \nabla \cdot \boldsymbol{\tau}, \\
\nabla \cdot \mathbf{U} & =0 .
\end{aligned}
$$

The corresponding equation for the dimensionless surfactant concentration $\Gamma$ on the interface is

$$
\frac{\partial \Gamma}{\partial t}+\nabla \cdot(\mathbf{U} \Gamma)=\frac{1}{\mathrm{Pe}} \nabla_{S}^{2} \Gamma+\Gamma \mathbf{n} \cdot \nabla \mathbf{U} \cdot \mathbf{n} .
$$

Here $C$ is a fractional volume function of the drop phase, $P$ denotes pressure, $\hat{\mathrm{g}}$ is a unit vector pointing in the direction of gravity, $\boldsymbol{\tau}$ is the viscous stress tensor, $\mathbf{F}_{\mathbf{S}}$ is the surface force arising from interfacial effects, $\nabla_{S}^{2}$ is the surface Laplacian, and $\mathbf{n}$ is a unit vector normal to the interface. The volume function $C$, which determines the interface position at any time, is advected with the local velocity $\mathbf{U}$. Equations (1)-(3) correspond to the flow of a mixture with variable properties (density $\rho$ and viscosity $\mu$ ), combined with advection of the volume fraction. The mixture takes the local fluid properties, with a volume fraction weighted average in computational cells containing the interface. The first term on the right of Equation (4) represents diffusion of surfactant on the interface while the second term describes the effect of changing interfacial area.

The velocity, length and time scales are chosen to be $(g a)^{1 / 2}, a$ and $(a / g)^{1 / 2}$, respectively. The resulting Reynolds, Weber, Froude and Peclet numbers in Equations (1)-(4) are:

$$
\mathrm{Re}=\frac{\rho_{r} g^{1 / 2} a^{3 / 2}}{\mu_{r}}, \quad \mathrm{We}=\frac{\rho_{r} g a^{2}}{\sigma_{e q}}, \quad \mathrm{Fr}=1, \quad \mathrm{Pe}=\frac{g^{1 / 2} a^{3 / 2}}{D} .
$$

where $\rho_{r}$ and $\mu_{r}$ denote reference values of density and viscosity, respectively, $D$ is the surface diffusivity of surfactant, and $\sigma_{e q}$ is the equilibrium 
coefficient of interfacial tension between the two liquids when the surfactant concentration is uniform $(\Gamma=1)$ over the interface. For this scaling, the Weber number is equivalent to the Bond number. Here, the density and viscosity of the column liquid are taken as reference values.

The relationship between the dimensionless interfacial tension and concentration is taken to be

$$
\sigma=\left(\frac{1+\beta}{1+\beta \Gamma}\right)^{3},
$$

as modelled by Borgas and Grotberg [2], where $\beta$ is a modelling parameter. This form extends the surface tension equation of state beyond the "close packed' region to all values of $\Gamma$, consistent with theoretical behaviour for large $\Gamma[2]$.

The explicit voF algorithm of Rudman [19] was used to solve Equations (1)-(3). The surface force $\mathbf{F}_{\mathbf{S}}$ is represented using Rudman's improved implementation of the CSF method [4]. The vof-based algorithm of James and Lowengrub [10] for surfactant transport was used to solve for surfactant surface concentration. A feature of the James and Lowengrub [10] algorithm is that Equation (4) for $\Gamma$ is not solved directly. Instead, the evolution of surface area $A$ and surfactant mass $M$ in computational cells is followed separately according to

$$
\begin{aligned}
& \frac{\partial A}{\partial t}+\nabla \cdot(\mathbf{U} A)=-A \mathbf{n} \cdot \nabla \mathbf{U} \cdot \mathbf{n}, \\
& \frac{\partial M}{\partial t}+\nabla \cdot(\mathbf{U} M)=\frac{A}{\mathrm{Pe}} \nabla_{S}^{2} \Gamma, \\
& \text { where } \quad \Gamma=\frac{M}{A} .
\end{aligned}
$$

The vof algorithm for advecting $C$ requires that the interface be reconstructed approximately as a line segment within an interface cell (using values of $C$ in surrounding cells). The surfactant concentration is assumed to have a linear dependence on distance along the line segment in each interface cell. 
Advection fluxes of $M$ and $A$ are evaluated by integrating along the part of the interface line segment that is advected out of the cell in each time step. Surfactant diffusion is implemented as a separate implicit update step. The method accurately conserves total surfactant mass.

James and Lowengrub [10] present and use a variety of simple problems with analytical solutions to test the different components of their algorithm (surface area and mass advection, changing surface area, surfactant diffusion). The present implementation, superimposed on the Rudman voF procedure, was also tested against those solutions to ensure the integrity of the resulting code. The calculation of the source term in Equation (7), representing surface area stretching, becomes inaccurate for the present problem, despite being accurate for a simpler test problem. So instead of explicitly including the source term in Equation (7), the surface area in each interface cell is set to equal the surface area generated by the reconstructed line segment therein at the beginning of each time step.

\section{Results and discussion}

Axi-symmetric flow calculations were performed in cylindrical polar coordinates on the symmetric half of a computational domain, having radius $2 a$ and length $24 a$, with 32 mesh cells spanning the drop radius $a$. A spherical drop is assumed to be released from rest on the centreline within an initially stationary liquid and then to move freely under the effect of gravity. Here the drop is taken to be less dense than the surrounding liquid, so it will rise. A free slip condition is imposed at the column walls, consistent with Davidson and coworkers $[6,7]$ who showed it was then possible to correct the predicted rise velocity for wall effects without using a computational domain of large radius.

A typical value of $\mathrm{Pe}$ for millimetre sized drops is about $10^{5}$, based on a diffusion coefficient $D=10^{-9} \mathrm{~m}^{2} / \mathrm{s}$ [2], so surfactant diffusion is negligi- 


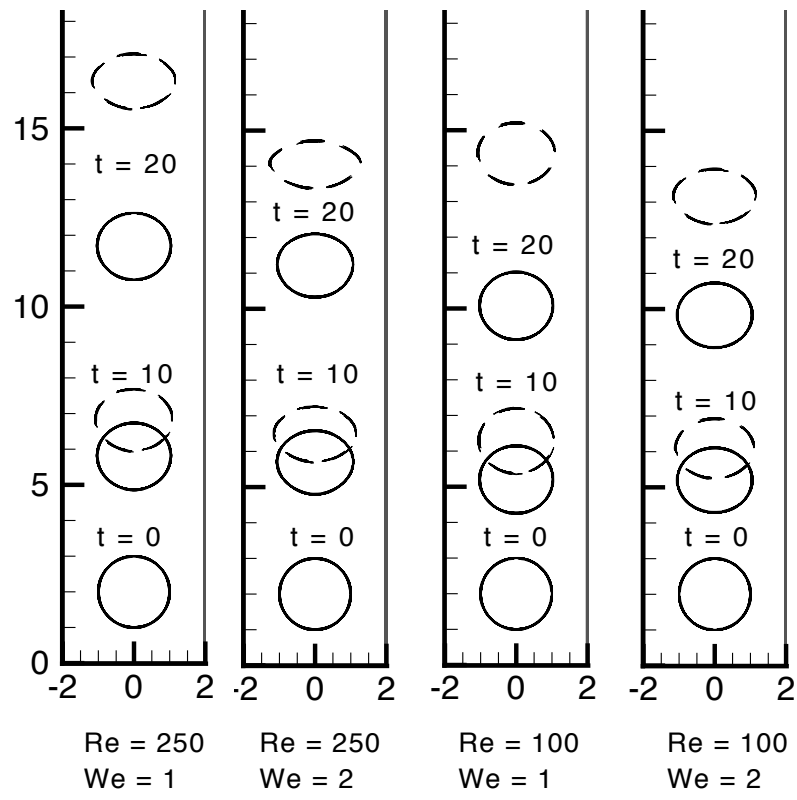

Figure 1: Evolution of drop shape with (solid lines) and without (dashed lines) surfactant transport.

ble here. However, it is desirable to choose a smaller Pe to reduce spatial oscillations in the surfactant concentration. Here we choose $\mathrm{Pe}=100$; little difference is obtained when we set $P e=1000$. Two values of Reynolds number $(\mathrm{Re}=100,250)$ and two values of Weber number $(\mathrm{We}=1,2)$ are considered. These represent a typical range for the drop rise experiments considered by Davidson and Stevens [7]. Drop/continuous phase density and viscosity ratios are taken to be 0.8 and 0.6 , respectively, which are also typical for the above experiments. The surface tension parameter is taken to be $\beta=0.03$. Increasing the mesh density by a factor of two in each direction resulted in very small differences in the predicted drop interface.

Figure 1 compares the evolution of drop shape for the four combinations of Reynolds and Weber numbers, with and without surfactant transport. When 
surfactant transport is ignored, the surfactant concentration (and hence the interfacial tension) is uniform on the interface. Steady rise conditions are not yet achieved for the results presented here. The results predict that surfactant transport reduces the drop rise velocity, consistent with current understanding. This occurs because Marangoni flows tend to immobilise the interface and retain a more spherical drop shape so that the drop rise is similar to that of a solid sphere. Also, increasing the Weber number from 1 to 2 (reducing interfacial tension) lowers the rise velocity when surfactant transport is absent because the drop is more deformed, and the interfacial area is larger, when $\mathrm{We}=2$. When surfactant transport occurs and the drop remains almost spherical, there is little difference in rise velocity for $\mathrm{We}=1,2$. Reducing the Reynolds number from 250 to 100 lowers the rise velocity regardless of surfactant transport, corresponding to an increase in viscosity.

The velocity field relative to the nose of the drop is shown in Figure 2 when $\operatorname{Re}=250$. Corresponding predictions for $\operatorname{Re}=100$ are qualitatively the same. For both values of We, surfactant transport has almost eliminated the internal recirculation within the drop so that there is very little momentum transfer from outside to inside the drop and the drop behaves more like a solid sphere. This phenomenon is known as 'surface hardening'. It occurs because the flow past the drop sweeps the surfactant towards the rear so that the interfacial tension there becomes less than at the front of the drop. In turn, this produces a (Marangoni) force tangential to the interface acting towards the front of the drop that opposes the internal recirculation. Note that the flow field for the surface-hardened drop exhibits a recirculating wake at the rear similar to that found at the rear of a solid sphere [18]. Also the drops without surfactant transport do not produce a wake, consistent with predictions of Dandy and Leal [5] for similar parameters.

Figures 3 and 4 illustrate the surfactant distribution on the interface when $\operatorname{Re}=100$. Both figures show that the surfactant is swept towards the bottom of the drop. Figure 3 demonstrates that the surfactant concen- 

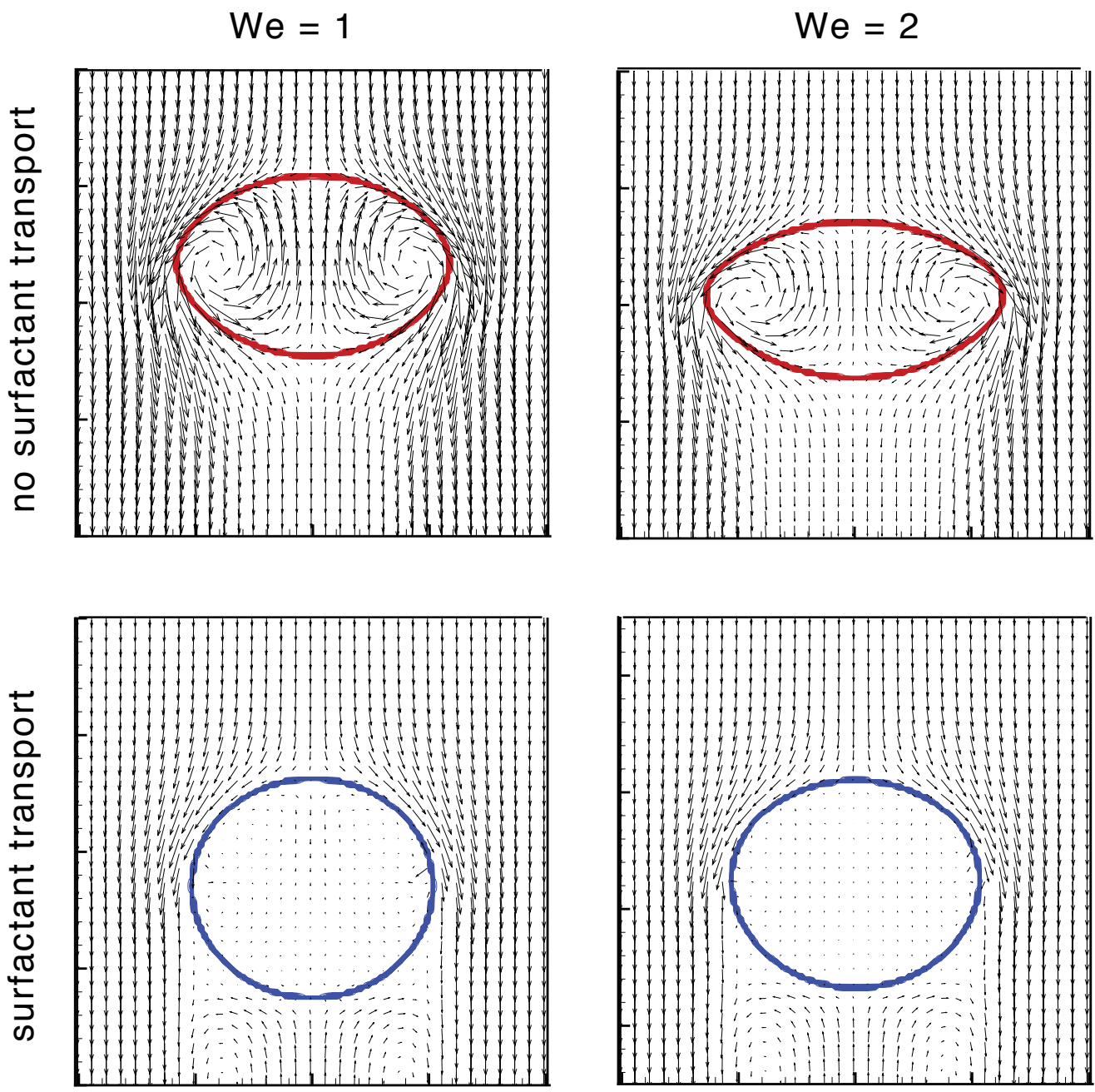

Figure 2: Velocity field relative to drop motion for $\mathrm{Re}=250$ with and without surfactant transport at time $t=20$. 

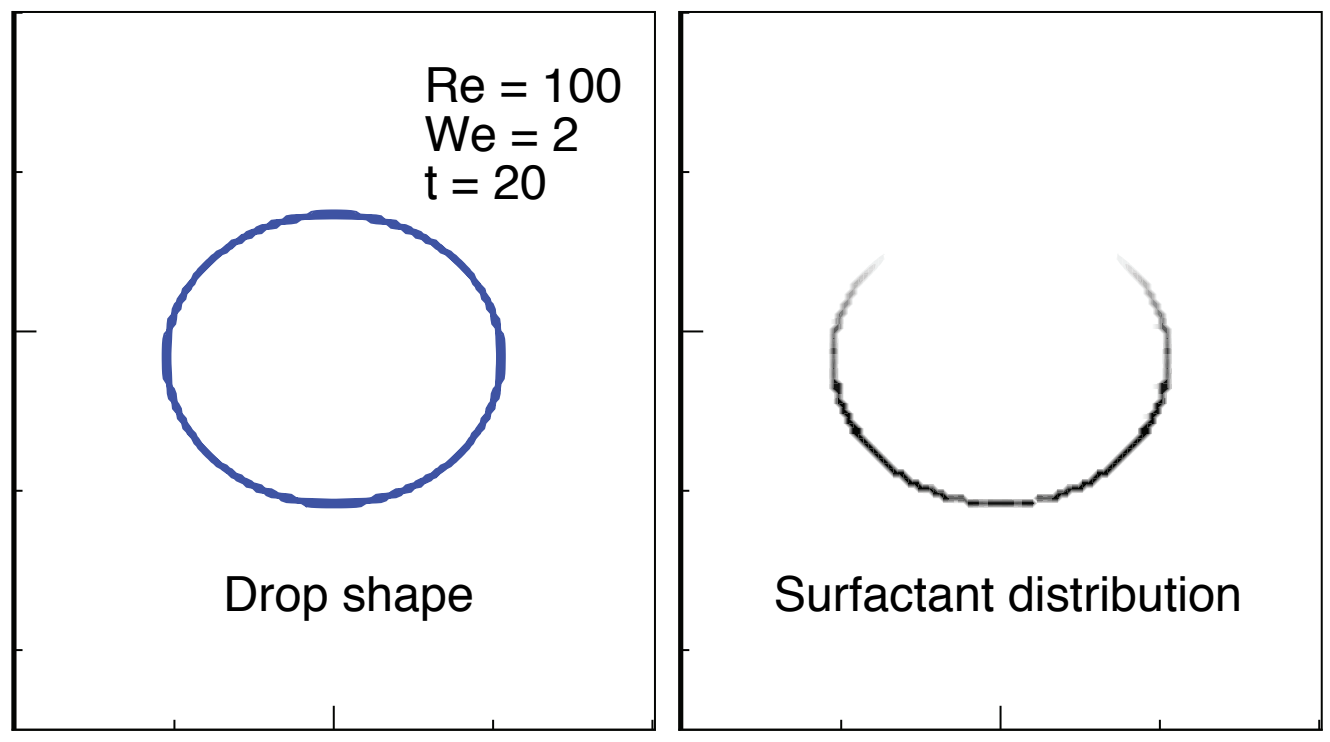

FiguRE 3: Example of drop shape and corresponding greyscale image of surfactant concentration. 

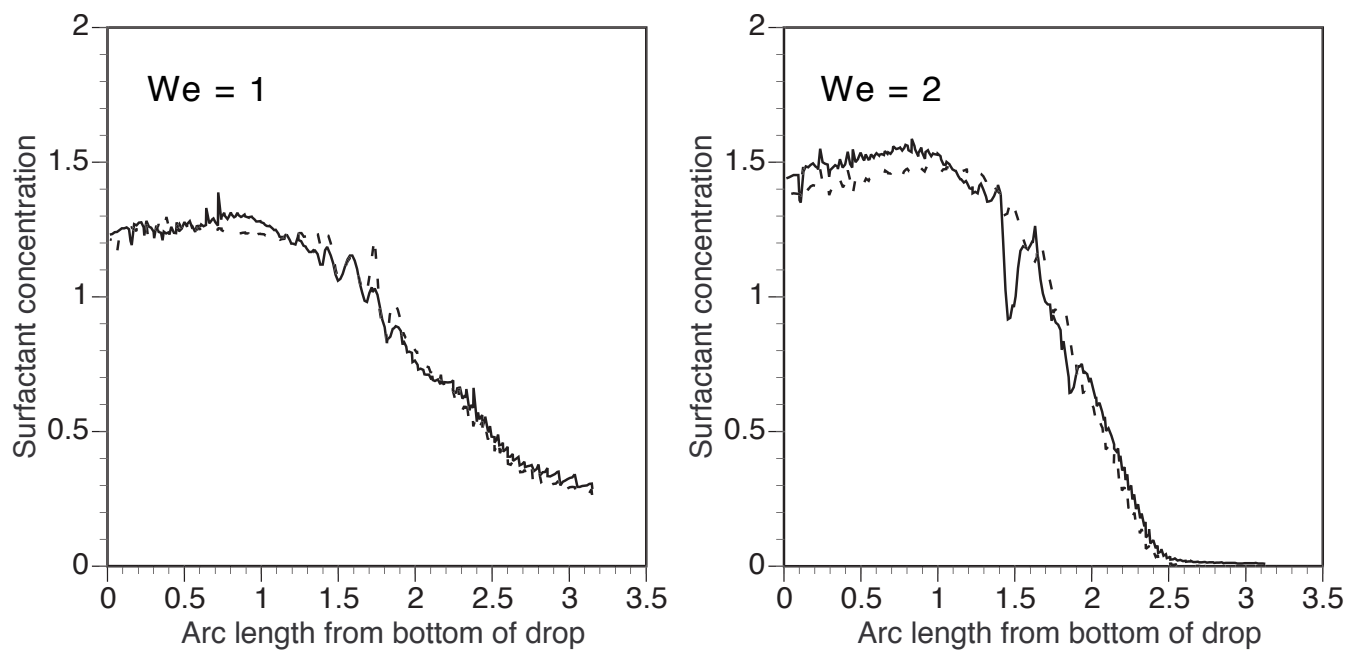

Figure 4: Surfactant concentration distribution for $\mathrm{Re}=100$ at $\mathrm{We}=1,2$ for $t=10$ (solid lines) and $t=20$ (dashed lines).

tration is restricted numerically to the interface as required. The wiggles in the surfactant distribution occur because the concentration is calculated using reconstructed interface line segments. Increasing the mesh density reduces the amplitude of the wiggles. The surface force is calculated using smoothed values of the (surfactant dependent) interfacial tension based on the smoothing kernel described by Rudman [19].

Figure 4 shows that the redistribution of surfactant towards the bottom half of the drop is less pronounced for $\mathrm{We}=1$ than it is for $\mathrm{We}=2$. This occurs because the interfacial tension is greater for $\mathrm{We}=1$ so that the Marangoni force, directed along the interface towards the front of the drop, is greater; this reduces the (downwards) liquid velocity at the interface relative to the drop motion. In the limit of sufficiently large interfacial tension, the liquid velocity at the interface becomes zero and the drop interface exhibits no slip like a solid surface. 


\section{Conclusion}

The vof numerical method of Rudman [19] has been extended to incorporate the VOF-based algorithm of James and Lowengrub [10] for transport of insoluble surfactant on the interface and the consequent development of Marangoni forces due to gradients in interfacial tension. The combined procedure is applied to a drop rising in a liquid column for Reynolds and Weber numbers typical of experiments reported in the solvent extraction literature. A reduction in interface mobility due to Marangoni forces, with an associated reduction in the drop rise velocity, is obtained as expected. The results confirm that the extended Rudman procedure can be used for the simulation of two fluid flow with interfaces in the presence of insoluble surfactant transport.

Acknowledgement This research was supported by the Australian Research Council Grants Scheme.

\section{References}

[1] I. B. Bazhlekov, P. D. Anderson, and H. E. H. Meijer. Numerical investigation of the effect of insoluble surfactants on drop deformation and breakup in simple shear flow. J. Colloid and Interface Science, 298:369-394, 2006. doi:10.1016/j.jcis.2005.12.017 C662

[2] M. S. Borgas and J. B. Grotberg. Monolayer flow on a thin film. J. Fluid Mech., 193:151-170, 1988. doi:10.1017/S0022112088002095 C665, C666

[3] J. M. Boulton-Stone. The effect of surfactant on bursting gas bubbles. J. Fluid Mech., 302:231-257, 1995. doi:10.1017/S0022112095004083 C663 
[4] J. U. Brackbill, D. B. Kothe, and C. Zemach. A continuum method for modelling surface tension. J. Comput. Phys., 100:335-354, 1992. doi:10.1016/0021-9991(92)90240-Y C665

[5] D. S. Dandy and L. G. Leal. Buoyancy-driven motion of a deformable drop through a quiescent liquid at intermediate Reynolds numbers. J. Fluid Mech., 208:161-192, 1989. doi:10.1017/S0022112089002818 $\mathrm{C} 668$

[6] M. R. Davidson and M. Rudman. Volume-of-Fluid calculation of heat or mass transfer across deforming interfaces in two-fluid flow. Numer. Heat Trans., Part B, 41(3/4):291-308, 2002. doi:10.1080/104077902753541023 C663, C666

[7] M. R. Davidson and G. W. Stevens. Mass transfer from deforming drops rising in a liquid column. Proc. International Solvent Extraction Conference, ISEC'05, pp. 1684-1693, 2005. C663, C666, C667

[8] M. A. Drumright-Clarke and Y. Renardy. The effect of insoluble surfactant at dilute concentration on drop breakup under shear with inertia. Phys. Fluids, 16(1):14-21, 2004. doi:10.1063/1.1628232 C662

[9] C. D. Eggleton, Y. P. Pawar and K. J. Stebe. Insoluble surfactants on a drop in an extensional flow: a generalization of the stagnated surface limit to deforming interfaces. J. Fluid Mech., 385:79-99, 1999. http: //journals. cambridge.org/action/displayAbstract?aid=14819 C662

[10] A. J. James and J. Lowengrub. A surfactant-conserving volume-of-fluid method for interfacial flows with insoluble surfactant. J. Comput. Phys., 201:685-722, 2004. doi:10.1016/j.jcp.2004.06.013 C662, C663, C665, C666, C672

[11] Y. W. Kruijt-Stegeman, F. N. van de Vosse and H. E. H. Meijer. Droplet behavior in the presence of insoluble surfactants. Phys. Fluids, 16(8):2785-2796, 2004. doi:10.1063/1.1756168 C662 
[12] J. Lee and C. Pozrikidis. Effect of surfactants on the deformation of drops and bubbles in Navier-Stokes flow. Computers and Fluids, 35:43-60, 2006. doi:10.1016/j.compfluid.2004.11.004 C662

[13] V. G. Levich and V. S. Krylov. Surface-tension-driven phenomena. Annu. Rev. Fluid Mech., 1:293-316, 1969. doi:10.1146/annurev.fl.01.010169.001453 C662

[14] X. Li and C. Pozrikidis. The effect of surfactants on drop deformation and on the rheology of dilute emulsions in Stokes flow. J. Fluid Mech., 341:165-194, 1997. http:

//journals . cambridge.org/action/displayAbstract?aid=13511 C662

[15] Y-C Liao, E. I. Franses and O. A. Basaran. Deformation and breakup of a stretching liquid bridge covered with an insoluble surfactant monolayer. Phys. Fluids, 18:022101-1-022101-21, 2006. doi:10.1063/1.2166657 C663

[16] Y. Pawar and K. J. Stebe. Marangoni effects on drop deformation in an extensional flow: The role of surfactant physical chemistry. I. Insoluble surfactants. Phys. Fluids, 8(7):1738-1751, 1996. doi:10.1063/1.868958 C662

[17] Y. Y. Renardy, M. Renardy and V. Cristini. A new volume-of-fluid formulation for surfactants and simulations of drop deformation under shear at a low viscosity ratio. Eur. J. Mech. B/Fluids, 21:49-59, 2002. doi:10.1016/S0997-7546(01)01159-1 C662

[18] Y. Rimon and S. I. Cheng. Numerical solution of a uniform flow over a sphere at intermediate Reynolds numbers. Phys. Fluids, 12(5):949-959, 1969. doi:10.1063/1.2163685 C668

[19] M. Rudman. A volume tracking method for incompressible multifluid flows with large density variations. Int. J. Numer. Meth. Fluids, 
28:357-378, 1998. doi:10.1002/(SICI)1097-

0363(19980815)28:2¡357::AID-FLD750¿3.0.CO;2-D C663, C665, C671, C672

[20] R. Scardovelli and S. Zaleski. Direct numerical simulation of free-surface and interfacial flow. Annu. Rev. Fluid Mech., 31:567-603, 1999. doi:10.1146/annurev.fluid.31.1.567 C663

[21] H. A. Stone and L. G. Leal. The effects of surfactants on drop deformation and breakup. J. Fluid Mech., 220:161-186, 1990. doi:10.1017/S0022112090003226 C662

[22] P. M. Vlahovska, M. Loewenbery and J. Blawzdziewicz. Deformation of a surfactant-covered drop in linear flow. Phys. Fluids, 17:103103-1-101103-18, 2005. doi:10.1063/1.2112727 C662 


\section{Author addresses}

1. M. R. Davidson, Department of Chemical and Biomolecular Engineering, The University of Melbourne, Parkville, Victoria 3010, Australia.

mailto:m.davidson@unimelb.edu.au

2. D. J. E. Harvie, Department of Chemical and Biomolecular Engineering, The University of Melbourne, Parkville, Victoria 3010, Australia. 